\title{
Is your hospital culturally safe?
}

I t's been almost six years since Brian Sinclair wheeled himself into the emergency room at Winnipeg's Health Sciences Centre seeking treatment for a blocked catheter. Thirty-four hours later staff checked on the Aboriginal amputee. Some had assumed Sinclair was just drunk or homeless. By that time, he was dead.

The final report of the inquest into Sinclair's death is expected soon. But presenters at Canada's first Indigenous Health Conference were dismayed that it will focus exclusively on patient flow.

"There was a level of pro-

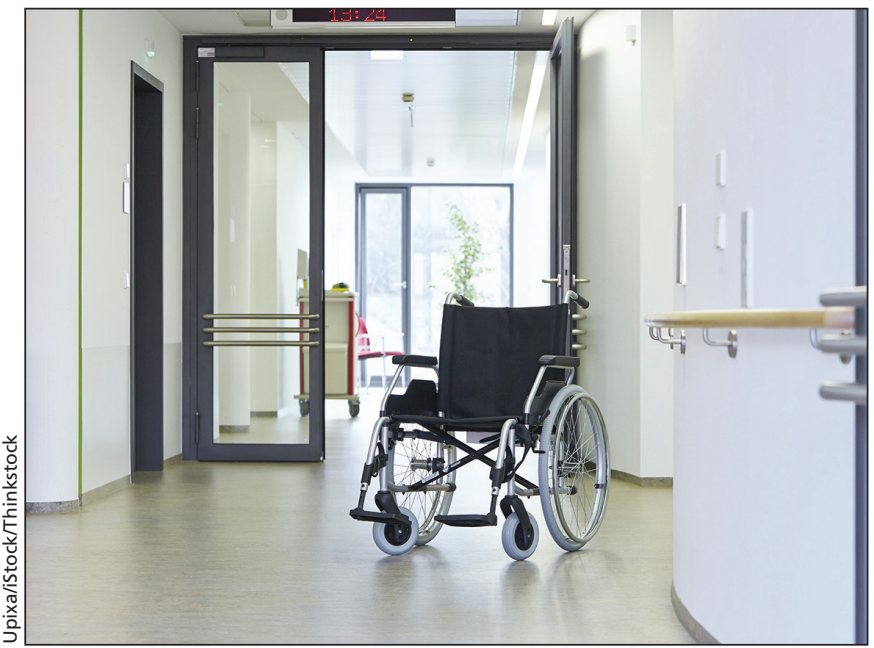

The case of Brian Sinclair, an Aboriginal man who died in a Winnipeg emergency room after waiting $\mathbf{3 4}$ hours in a wheelchair, has spurred discussion about cultural safety. filing and stereotyping that had to happen in order for a physically disabled man to languish and die in full public view and surrounded by health professionals," said Vanessa Ambtman-Smith, Aboriginal health lead for Ontario's South West Local Health Integration Network (LHIN). "We won't have an opportunity to further pursue some of that underlying racism.'

Too often in health care the r-word "gets couched in terms of stereotypes, bias, discrimination and health inequities," she said. "This is what it looks like when the system is failing; this is an example of an environment that is not culturally safe."

\section{What is cultural safety?}

Cultural safety was a new concept for some at the Toronto conference held Nov. 20 and 21. Until recently, most Aboriginal health initiatives targeting health workers have focused more on raising cultural awareness. Often that boils down to learning about differences in the beliefs, practices and needs of Indigenous groups, as well as the historic roots of health disparities.

Cultural safety is what you do with that knowledge - whether that means examining your own biases or challeng- ing those of a health institution or system. More importantly, "it's an outcome that's defined and experienced by those who receive the service," explained Ambtman-Smith. In other words, if an environment doesn't feel safe, it's not.

The concept originated in New Zealand, but is steadily gaining traction in Canada. It's included in mandatory Indigenous cultural competency training in British Columbia under the province's Tripartite First Nations Health Plan introduced in 2007. To date, more than 17000 people have completed the training. Now Ontario has developed its own version of the training, which some 1000 individuals have completed since June.

Self-reflection to challenge underlying assumptions is a major part of the eight-week online course, said Guy Hagar, an Indigenous cultural competency trainer with the Southwest Ontario Aboriginal Health Access Centre. "When we evaluate ourselves we tend to place ourselves higher on the ladder [of cultural sensitivity] than we really are."

\section{Cultural safety in maternal care}

Cultural safety is also about actively inviting and creating a space for cul- tural expression within health care, said Toronto midwife Sara Wolfe, in a presentation on behalf of the Society of Obstetricians and Gynecologists of Canada.

For example, "we often hear that people don't think they can smudge - burn traditional medicines - in a hospital, but many hospitals are now more supportive of incorporating that into a birth plan or even into prenatal appointments."

The onus is on caregivers to ask families if there are special traditions or practices they want to include in care, and offer examples, Wolfe added. She shared the story of an Aboriginal mother who was quick to say she wasn't traditional when asked generally about cultural practices.

"So I asked her about smudging at birth, about keeping the placenta, welcoming ceremonies, cedar baths ... and she wanted all of them," said Wolfe. "Either she didn't know she could ask for these things or didn't even see them as distinct culture practices."

There has been pushback against what some health workers view as special treatment when requests go against hospital policies - for example, women requesting that a large number of family members be present at a birth. "We do have nurses saying, 'The midwives just do whatever they want to do,' but I would advocate for that for a family ... even if they are non-Indigenous," said Wolfe.

But particularly in the case of Indigenous women, "this is about a constitutional right to be able to practise our traditional ways, and if someone wants to reclaim birth as part of their healing process, then a hospital policy is not going to stop me from advocating." Lauren Vogel, CMAJ

CMAJ 2015. DOI:10.1503/cmaj.109-4953 